\title{
Classification of algebras with minimal quadratic growth of identities
}

\author{
A. C. Vieira \\ Departamento de Matemática, Instituto de Ciências Exatas, \\ Universidade Federal de Minas Gerais, 30123-970, Brasil \\ E-mail address: anacris@ufmg.br
}

\section{S. M. Alves Jorge}

Departamento de Matemática, Pontifícia Universidade Católica de Minas Gerais, Belo Horizonte, 30535-610, Brasil

E-mail address: sandramara@pucminas.br

\begin{abstract}
The main goal of this paper is to prove that the five algebras which were used in [3] to classify (up to PI-equivalence) the algebras whose sequence of codimensions is bounded by a linear function generate the only five minimal varieties of quadratic growth.
\end{abstract}

\section{Introduction}

Throughout this paper we shall denote by $F$ a field of characteristic zero and $A$ an associative algebra over $F$. We denote by $F\langle X\rangle$ the free associative algebra on the countable set $X=\left\{x_{1}, x_{2}, \ldots\right\}$ and $\operatorname{Id}(A)=\{f \in$ $F\langle X\rangle \mid f \equiv 0$ on $A\}$ the $T$-ideal of all identities satisfied by $A$. Also denote by $\left\langle f_{1}, \cdots, f_{n}\right\rangle_{T}$ the $T$-ideal generated by the polynomials $f_{1}, \cdots, f_{n} \in$ $F\langle X\rangle$.

Let $P_{n}$ be the $F$-vector space of multilinear polynomials in $x_{1}, \ldots, x_{n}$ that is, $P_{n}=\operatorname{span}_{F}\left\{x_{\sigma(1)} \cdots x_{\sigma(n)} \mid \sigma \in S_{n}\right\}$. We can consider the following left action of the symmetric group $S_{n}$ on $P_{n}$ given by

$$
\sigma f\left(x_{1}, \cdots, x_{n}\right)=f\left(x_{\sigma(1)}, \cdots, x_{\sigma(n)}\right),
$$

where $\sigma \in S_{n}$ and $f\left(x_{1}, \cdots, x_{n}\right) \in P_{n}$ and note that the subspace $P_{n} \cap \operatorname{Id}(A)$ is invariant under this action. 
Hence

$$
P_{n}(A)=\frac{P_{n}}{P_{n} \cap \operatorname{Id}(A)}
$$

has a structure of $S_{n}$-module and its corresponding $S_{n}$-character, denoted by $\chi_{n}(A)$, is called the $n$th cocharacter of $A$. We define $c_{n}(A)=$ $\operatorname{dim}_{F} P_{n}(A)$, that is $c_{n}(A)=\operatorname{deg} \chi_{n}(A)$ is the $n$th codimension of $A$.

The behavior of the sequence of codimensions of an $F$-algebra $A$ has been persistently studied and since $c_{n}(A)=\operatorname{dim}_{F} P_{n}(A)$ it is clear that $c_{n}(A) \leq n$ !, but in case $A$ is a PI-algebra, a famous theorem of Regev [7] asserts that $c_{n}(A)$ is exponentially bounded. In [4] Kemer proved that $c_{n}(A)$ is polynomially bounded if and only if $G, U T_{2} \notin \operatorname{var}(A)$, where $\operatorname{var}(A)$ denotes the variety of algebras generated by $A$ and $G$ is the infinite dimensional Grassmann algebra, while $U T_{2}$ is the $2 \times 2$ upper triangular matrix algebra over $F$.

Since $\operatorname{char} F=0$, the representation theory of the symmetric group becomes a powerful tool in the study of the sequence $c_{n}(A)$ and it is a well known fact that the irreducible $S_{n}$-characters are in one-to-one correspondence with the partitions $\lambda$ of $n$. Thus, by complete redutibility, we can write $\chi_{n}(A)=\sum_{\lambda \vdash n} m_{\lambda} \chi_{\lambda}$, where $\chi_{\lambda}$ is the irreducible $S_{n}$-character associated to $\lambda \vdash n$ and $m_{\lambda} \geq 0$ is the corresponding multiplicity.

It is clear that, by taking degrees, we get $c_{n}(A)=\sum_{\lambda \vdash n} m_{\lambda} d_{\lambda}$, where $d_{\lambda}=\chi_{\lambda}(1)$ can be explicited by the hook formula [5]. We also have $l_{n}(A)=$ $\sum_{\lambda \vdash n} m_{\lambda}$ called the $n$th colength of $A$.

Kemer's result can also be established in an equivalent way as a problem about representations of the symmetric group as follows (see [6] and [1]): $c_{n}(A)$ is polynomially bounded if and only if $l_{n}(A) \leq k$, for some constant $k$ and for all $n \geq 1$.

It seems to be interesting to give a characterization of algebras $A$ with a particular property of the sequence $c_{n}(A), n=1,2, \cdots$, by avoiding some algebras in $\operatorname{var}(A)$. In [3] Giambruno and La Mattina exhibit a list of seven algebras, named $M_{i}$, for $1 \leq i \leq 7$, to be excluded from the variety of an algebra $A$ over a field $F$ of characteristic zero such that the sequence of codimensions $c_{n}(A), n=1,2, \cdots$, has linear or constant growth. For that they studied the behavior of the sequences of codimensions $c_{n}\left(M_{i}\right)$, for $1 \leq i \leq 7$. Complete results were given when $1 \leq i \leq 6$ but for $M_{7}$ the only conclusion was that its sequence of codimensions is bounded from below by $n^{2}$.

Our main goal here is to compute the codimensions, the cocharacters and the colengths of $M_{7}$ by giving a complete description of the $T$-ideal of $M_{7}$ and a basis of $\frac{P_{n}}{P_{n} \cap I d\left(M_{7}\right)}$ over $F$. As a consequence we classify all minimal 
varieties of quadratic growth which are varieties whose codimensions have quadratic growth and all proper subvarieties have linear or constant growth.

We will see that the representation theory of the general linear group will be efficiently used to compute some polynomial identities in the next section. For we introduce the free associative algebra in $m$ variables $F_{m}\langle X\rangle=$ $F\left\langle x_{1}, \cdots, x_{m}\right\rangle$ and $\mathcal{U}=\operatorname{span}_{F}\left\{x_{1}, \cdots, x_{m}\right\}$. We have a natural left action of $G L(\mathcal{U}) \cong G L_{m}$ on the space $\mathcal{U}$ which can be diagonally extended on $F_{m}\langle X\rangle$.

In this way the subspace $F_{m}\langle X\rangle \cap \operatorname{Id}(A)$ is invariant under this action, hence

$$
F_{m}(A)=\frac{F_{m}\langle X\rangle}{F_{m}\langle X\rangle \cap \operatorname{Id}(A)}
$$

has a structure of $G L_{m}$-module and we also consider its $G L_{m}$-submodule

$$
F_{m}^{n}(A)=\frac{F_{m}^{n}\langle X\rangle}{F_{m}^{n}\langle X\rangle \cap I d(A)},
$$

where $F_{m}^{n}\langle X\rangle$ is the space of homogeneous polynomials of degree $n$ in the variables $x_{1}, \cdots, x_{m}$. We denote by $\psi_{n}(A)$ the $G L_{m}$-character of $F_{m}^{n}(A)$ and write $\psi_{n}(A)=\sum_{\lambda \vdash n} \tilde{m}_{\lambda} \psi_{\lambda}$, where $\psi_{\lambda}$ is the irreducible $G L_{m}$-character associated to the partition $\lambda \vdash n$ and $\tilde{m}_{\lambda} \geq 0$ is the corresponding multiplicity.

Some important features about the multiplicities $\tilde{m}_{\lambda}$ of the $G L_{m}$-character $\psi_{\lambda}$ which are related to the multiplicities $m_{\lambda}$ of the $S_{n}$-character $\chi_{\lambda}$ are listed in the following remarks, where $h_{i}(\lambda)$ denotes the height of the $i$ th column of the Young diagram of $\lambda$.

Remark 1.1 ([2], Theorem 12.4.20). For all $\lambda \vdash n$ such that $h_{1}(\lambda) \leq m$ we have $m_{\lambda}=\tilde{m}_{\lambda}$.

Remark 1.2 ([2], Theorem 12.4.12). Any irreducible submodule of $F_{m}^{n}(A)$ corresponding to the partition $\lambda=\left(\lambda_{1}, \cdots, \lambda_{t}\right)$ of $n$ is generated by a nonzero polynomial of the form

$$
f_{\lambda}=\prod_{i=1}^{\lambda_{1}} S t_{h_{i}(\lambda)}\left(x_{1}, \cdots, x_{h_{i}(\lambda)}\right) \sum_{\sigma \in S_{n}} \alpha_{\sigma} \sigma,
$$

where $\alpha_{\sigma} \in F$, the right action of $S_{n}$ on $F_{m}^{n}(A)$ is defined as place permutation and $S t_{q}\left(x_{1}, \cdots, x_{q}\right)$ denotes the well known standard polynomial of degree $q$.

The polynomial $f_{\lambda}$ is called highest weight vector associated to $\lambda$. Furthermore, if $T_{\lambda}$ is a standard Young tableau, by considering the only permutation $\sigma \in S_{n}$ such that the integers $\sigma(1), \cdots, \sigma\left(h_{1}(\lambda)\right)$, in this order, fill in the first column of $T_{\lambda}$ from the top to bottom, the integers 
$\sigma\left(h_{1}(\lambda)+1\right), \cdots, \sigma\left(h_{1}(\lambda)+h_{2}(\lambda)\right)$ fill in the second column of $T_{\lambda}$, etc, we define

$$
f_{T_{\lambda}}=\prod_{i=1}^{\lambda_{1}} S t_{h_{i}(\lambda)}\left(x_{1}, \cdots, x_{h_{i}(\lambda)}\right) \sigma^{-1},
$$

to be the highest weight vector associated to the standard tableau $T_{\lambda}$.

Remark 1.3 ([2], Proposition 12.4.14). Each polynomial $f_{\lambda}$ can be expressed uniquely as a linear combination of polynomials $f_{T_{\lambda}}$.

From the remarks above it follows an important and useful fact.

Remark 1.4. The multiplicity $\tilde{m}_{\lambda} \neq 0$ if and only if there is a standard Young tableau $T_{\lambda}$ corresponding to $\lambda$ such that $f_{T_{\lambda}} \notin I d(A)$. Moreover $\tilde{m}_{\lambda}$ is equal to the maximal number of linearly independent highest weight vectors $f_{T_{\lambda}}$ in $F_{m}^{n}(A)$.

\section{The PI-algebra $M_{7}$}

In [3] Giambruno and La Mattina introduce a list of PI-algebras which play a fundamental role in the classification of the algebras $A$ with slow codimension growth i.e., $c_{n}(A) \leq k n$, for all $n$ and some constant $k$, or $c_{n}(A) \leq k$, for all $n$ and some constant $k$. The algebras in this list follow below.

$$
\begin{gathered}
M_{1}=\left(\begin{array}{cc}
F & F \\
0 & 0
\end{array}\right), M_{2}=\left(\begin{array}{cc}
0 & F \\
0 & F
\end{array}\right), M_{3}=\left\{\left(\begin{array}{ccc}
a & b & c \\
0 & a & d \\
0 & 0 & a
\end{array}\right) \mid a, b, c, d \in F\right\}, \\
M_{4}=\left(\begin{array}{ccc}
F & F & F \\
0 & 0 & F \\
0 & 0 & 0
\end{array}\right), M_{5}=\left(\begin{array}{ccc}
0 & F & F \\
0 & 0 & F \\
0 & 0 & F
\end{array}\right), M_{6}=\left(\begin{array}{ccc}
0 & F & F \\
0 & F & F \\
0 & 0 & 0
\end{array}\right), \\
\text { and } M_{7}=\left\{\left(\begin{array}{lll}
a & b & c \\
0 & 0 & d \\
0 & 0 & a
\end{array}\right) \mid a, b, c, d \in F\right\} .
\end{gathered}
$$

For some those algebras they proved ([3], Lemmas 4 and 6) the following.

Lemma 2.1. For all $n>3$, we have

(1) $\operatorname{Id}\left(M_{3}\right)=\langle[x, y, z],[x, y][z, w]\rangle_{T}$ and $c_{n}\left(M_{3}\right)=\frac{n(n-1)+2}{2}$

(2) $\operatorname{Id}\left(M_{4}\right)=\langle[x, y] z w\rangle_{T}$ and $c_{n}\left(M_{4}\right)=n(n-1)$

(3) $\operatorname{Id}\left(M_{5}\right)=\langle z w[x, y]\rangle_{T}$ and $c_{n}\left(M_{5}\right)=n(n-1)$

(4) $\operatorname{Id}\left(M_{6}\right)=\langle z[x, y] w\rangle_{T}$ and $c_{n}\left(M_{6}\right)=n(n-1)$

But Giambruno and La Mattina had just proved that there is an irreducible $S_{n^{-}}$-character appearing with non-zero multiplicity in $\chi_{n}\left(M_{7}\right)$ whose degree is $\geq n^{2} / 2$ and concluded that the codimensions of $M_{7}$ grow asymptotically at least as $n^{2} / 2$. 
In this section we present a complete description of the $T$-ideal $\operatorname{Id}\left(M_{7}\right)$ and of the sequence of codimensions $\left\{c_{n}\left(M_{7}\right)\right\}_{n \geq 1}$. As a consequence we determine the cocharacters $\chi_{n}\left(M_{7}\right)$ and the colengths $l_{n}\left(M_{7}\right)$, for $n \geq 1$. Our main result is the following.

Theorem 2.2. For any $n \geq 4$ we have

(1) $\chi_{n}\left(M_{7}\right)=\chi_{(n)}+2 \chi_{(n-1,1)}+\chi_{(n-2,1,1)}+\chi_{(n-2,2)}$

(2) $l_{n}\left(M_{7}\right)=5$

(3) $X=T(I) \cup T(I I) \cup T(I I I) \cup T(I V)$ is a basis of $P_{n}\left(\bmod P_{n} \cap\right.$ $\left.\operatorname{Id}\left(M_{7}\right)\right)$

(4) $c_{n}\left(M_{7}\right)=n(n-1)$

(5) $\operatorname{Id}\left(M_{7}\right)=\left\langle f_{1}, f_{2}, f_{3}, f_{4}, f_{5}, f_{6}\right\rangle_{T}$.

Where we are denoting the set of polynomials of type $L$ by $T(L)$ defined as follows.

Polynomial of Type $\mathbf{I}-|T(I)|=1$.

$$
P_{I}=x_{1} x_{2} \cdots x_{n} \text {. }
$$

Polynomials of Type II - $|T(I I)|=n-1$.

$$
\begin{aligned}
& P_{I I}^{(2)}=w\left(x_{3}\left[x_{2}, x_{1}\right] x_{4}-x_{3} x_{4}\left[x_{2}, x_{1}\right]\right) \\
& P_{I I}^{(3)}=w\left(x_{2}\left[x_{3}, x_{1}\right] x_{4}-x_{2} x_{4}\left[x_{3}, x_{1}\right]\right) \\
& P_{I I}^{(4)}=w\left(x_{2}\left[x_{4}, x_{1}\right] x_{3}-x_{2} x_{3}\left[x_{4}, x_{1}\right]\right)
\end{aligned}
$$

If $n \geq 5, P_{I I}^{(j)}=w\left(x_{3}\left[x_{j}, x_{1}\right] x_{4}-x_{3} x_{4}\left[x_{j}, x_{1}\right]\right)$, for $5 \leq j \leq n$.

Polynomials of Type III - $|T(I I I)|=n-1$.

$$
P_{I I I}^{(j)}=w\left(\left[x_{j}, x_{1}\right] x_{i_{1}} \cdots x_{i_{n-2}}-x_{i_{1}}\left[x_{j}, x_{1}\right] x_{i_{2}} \cdots x_{i_{n-2}}\right), \text { for } 2 \leq j \leq n
$$

where $i_{1}<i_{2}<\cdots<i_{n-2}$.

Polynomials of Type IV - $|T(I V)|=n^{2}-3 n+1$.

For $5 \leq j \leq n$,

$$
\begin{aligned}
P_{2,1,3,4} & =w\left(\left[x_{2}, x_{1}\right]\left[x_{3}, x_{4}\right]\right) \\
\tilde{P}_{2,1,3,4} & =w\left(\left[x_{3}, x_{4}\right]\left[x_{2}, x_{1}\right]\right) \\
P_{3,1,2,4} & =w\left(\left[x_{3}, x_{1}\right]\left[x_{2}, x_{4}\right]\right) \\
P_{4,1,2,3} & =w\left(\left[x_{4}, x_{1}\right]\left[x_{2}, x_{3}\right]\right) \\
\tilde{P}_{4,1,2,3} & =w\left(\left[x_{2}, x_{3}\right]\left[x_{4}, x_{1}\right]\right)
\end{aligned}
$$

$$
P_{j, 1,3,4}=w\left(\left[x_{j}, x_{1}\right]\left[x_{3}, x_{4}\right]\right)
$$




$$
\begin{aligned}
\tilde{P}_{j, 1,3,4} & =w\left(\left[x_{3}, x_{4}\right]\left[x_{j}, x_{1}\right]\right) \\
P_{2,1, j-1, j} & =w\left(\left[x_{2}, x_{1}\right]\left[x_{j-1}, x_{j}\right]\right) \\
\tilde{P}_{2,1, j-1, j} & =w\left(\left[x_{j-1}, x_{j}\right]\left[x_{2}, x_{1}\right]\right)
\end{aligned}
$$

and finally,

$$
\begin{aligned}
P_{i, 1,2, j} & =w\left(\left[x_{i}, x_{1}\right]\left[x_{2}, x_{j}\right]\right) \\
\tilde{P}_{i, 1,2, j} & =w\left(\left[x_{2}, x_{j}\right]\left[x_{i}, x_{1}\right]\right),
\end{aligned}
$$

where $4 \leq i \leq j-1$.

Furthermore the identities $f_{i} ; 1 \leq i \leq 6$ are defined as below.

$$
\begin{aligned}
& f_{1}=[x, y][z, w]+[z, w][x, y]+[y, z][x, w]+ \\
& {[x, w][y, z]+[z, x][y, w]+[y, w][z, x]=S t_{4}(x, y, z, w)} \\
& f_{2}=[x, y][z, w]+[z, w][x, y]+x[z, w] y-y[z, w] x \\
& f_{3}=2 x y[w, z]+w x[z, y]+w y[z, x]+z x[y, w]+z y[x, w]+ \\
& {[x, y][z, w]+[x, z][w, y]-[w, y][x, z]+[y, z][w, x]-[w, x][y, z]} \\
& f_{4}=[x, y] z[w, t], f_{5}=[[x, y][z, w], t], \text { and } f_{6}=[z[x, y] w, t] .
\end{aligned}
$$

In order to prove the theorem above we make some remarks and prove some lemmas.

It is not difficult to see that $f_{1}, f_{2}, f_{3}, f_{4}, f_{5}, f_{6}$ belong to $\operatorname{Id}\left(M_{7}\right)$. It is interesting to note that $f_{4}, f_{5}, f_{6}$ were obtained from the structure of the PI-algebra $M_{7}$, but we got the identities of degree 4 for $M_{7}$ from the highest weight vectors associated to partitions of $n=4$.

Applying the multilinearization method to some obvious identities of $M_{7}$, using commutator calculus and some of the identities above, we get

$$
\begin{gathered}
z[x, w] y+y[x, z] w+w[x, y] z \equiv 0 \text { in } M_{7} \\
(x y[z, w]-x[z, w] y)=(x z[y, w]-x[y, w] z)-(x w[y, z]-x[y, z] w) \\
x[y, z][w, t] \equiv y[x, z][w, t]-z[x, y][w, t] \\
x[y, z][w, t] \equiv w[y, z][x, t]-t[y, z][x, w] \\
x[z, w] y-x y[z, w] \equiv y[z, w] x-y x[z, w]-2[x, y][z, w]-[z, w][x, y] .
\end{gathered}
$$

In the next results we consider $Q=\left\langle f_{1}, f_{2}, f_{3}, f_{4}, f_{5}, f_{6}\right\rangle_{T}$. 
Lemma 2.3. For $n=4$, any polynomial of the form $x_{r}\left[x_{k}, x_{1}\right] x_{s}-x_{r} x_{s}\left[x_{k}, x_{1}\right]$ with $r, s, k \in\{2,3,4\}$ can be written, modulo $Q$, as a linear combination of polynomials of types II and $I V$ over $F$.

Proof. First we note that the element $\left[x_{4}, x_{2}\right]\left[x_{3}, x_{1}\right]$ is a combination of elements of type IV, since $f_{1}=S t_{4} \in Q$. Now, using that $f_{2} \in Q$ we have

$$
x[z, w] y-x y[z, w] \equiv y[z, w] x-y x[z, w]-2[x, y][z, w]-[z, w][x, y] .
$$

Then $\left[x_{4}\left[x_{2}, x_{1}\right] x_{3}-x_{4} x_{3}\left[x_{2}, x_{1}\right] \equiv\right.$

$$
x_{3}\left[x_{2}, x_{1}\right] x_{4}-x_{3} x_{4}\left[x_{2}, x_{1}\right]-2\left[x_{4}, x_{3}\right]\left[x_{2}, x_{1}\right]-\left[x_{2}, x_{1}\right]\left[x_{4}, x_{3}\right] .
$$

Now applying an analogous reasoning for $x_{4}\left[x_{3}, x_{1}\right] x_{2}-x_{4} x_{2}\left[x_{3}, x_{1}\right]$ and $x_{3}\left[x_{4}, x_{1}\right] x_{2}-x_{3} x_{2}\left[x_{4}, x_{1}\right]$, we conclude the lemma.

In the next result we consider the polynomials $P_{k, l, r, s}=w\left[x_{k}, x_{l}\right]\left[x_{r}, x_{s}\right]$ and $\tilde{P}_{k, l, r, s}=w\left[x_{r}, x_{s}\right]\left[x_{k}, x_{l}\right]$ where $w=x_{i_{1}} \cdots x_{i_{m}}$ with $i_{1}<\cdots<i_{m}$ and $i_{1}, \cdots, i_{m} \notin\{k, l, r, s\}$.

Lemma 2.4. For all $n \geq 4$, any polynomial $P_{k, l, r, s}$ (and $\tilde{P}_{k, l, r, s}$ ) can be written, modulo $Q$, as a linear combination over $F$ of elements in $T(I V)$, where $l, r, s, k \in\{1, \cdots, n\}$.

Proof. First we consider $l=1$ and prove the result by induction on $n$. By the proof of Lemma 2.3, the result is true for $n=4$. Hence we may assume $n \geq 5$ and suppose that the result is valid for $n-1$.

We work with the polynomials $P_{k, 1, r, s}$, by splitting the proof in three cases. In all of them we consider $w^{\prime}$ the monomial in the remaining ordered variables.

Case 1: $r, s, k \in\{2, \cdots, n-1\}$.

In this case we have $P_{k, 1, r, s}=w\left(\left[x_{k}, x_{1}\right]\left[x_{r}, x_{s}\right]\right)$ where $w$ is a monomial containing the variable $x_{n}$. Therefore, we can write

$$
P_{k, 1, r, s} \equiv x_{n}\left(w^{\prime}\left[x_{k}, x_{1}\right]\left[x_{r}, x_{s}\right]\right)
$$

and using the induction hypothesis and the fact that we can reorganize the variables that are not in the commutators, the result follows.

Case 2: $s=n$.

We have $P_{k, 1, r, n}=w\left(\left[x_{k}, x_{1}\right]\left[x_{r}, x_{n}\right]\right)$ and let us consider the possibilities for $k$. First we assume $k=2$. Clearly the result follows for $r=n-1$ since $P_{2,1, n-1, n}$ belongs to $T(I V)$. On the other hand, if $r \neq n-1$ by using (3) we write

$$
\begin{aligned}
P_{2,1, r, n} & \equiv w^{\prime} x_{n-1}\left(\left[x_{2}, x_{1}\right]\left[x_{r}, x_{n}\right]\right) \\
& \equiv w^{\prime} x_{r}\left[x_{2}, x_{1}\right]\left[x_{n-1}, x_{n}\right]-w^{\prime} x_{n}\left[x_{2}, x_{1}\right]\left[x_{n-1}, x_{r}\right]
\end{aligned}
$$

and now the result follows from Case 1. 
Thus let us suppose $4 \leq k \leq n-1$. For $r=2$, we have $P_{k, 1,2, n}$ is already in $T(I V)$ and for $r \neq 2$ we have

$$
\begin{aligned}
P_{k, 1, r, n} & \equiv w^{\prime} x_{2}\left(\left[x_{k}, x_{1}\right]\left[x_{r}, x_{n}\right]\right) \\
& \equiv w^{\prime} x_{r}\left[x_{k}, x_{1}\right]\left[x_{2}, x_{n}\right]-w^{\prime} x_{n}\left[x_{k}, x_{1}\right]\left[x_{2}, x_{r}\right]
\end{aligned}
$$

and use again the first case.

Finally, when $k=3$, for $r=2$ we use (4) and obtain

$$
\begin{aligned}
P_{3,1,2, n} & \equiv w^{\prime} x_{4}\left(\left[x_{3}, x_{1}\right]\left[x_{2}, x_{n}\right]\right) \\
& \equiv w^{\prime} x_{3}\left[x_{4}, x_{1}\right]\left[x_{2}, x_{n}\right]-w^{\prime} x_{1}\left[x_{3}, x_{4}\right]\left[x_{2}, x_{n}\right]
\end{aligned}
$$

and since $w^{\prime} x_{1}\left[x_{3}, x_{4}\right]\left[x_{2}, x_{n}\right]=w^{\prime} x_{2}\left[x_{3}, x_{4}\right]\left[x_{1}, x_{n}\right]-w^{\prime} x_{n}\left[x_{3}, x_{4}\right]\left[x_{2}, x_{n}\right]$ we are done and we can consider $r \neq 2$, once more we apply the same ideas of Case 1.

Case 3: $k=n$.

In this case, we have $2 \leq r, s \leq n-1$ and $P_{n, 1, r, s} \equiv w^{\prime} x_{j}\left[x_{n}, x_{1}\right]\left[x_{r}, x_{s}\right]$ for some $j<n$. Hence

$$
P_{n, 1, r, s} \equiv w^{\prime} x_{n}\left[x_{j}, x_{1}\right]\left[x_{r}, x_{s}\right]-w^{\prime} x_{1}\left[x_{j}, x_{n}\right]\left[x_{r}, x_{s}\right]
$$

and using that $x_{1}\left[x_{j}, x_{n}\right]\left[x_{r}, x_{s}\right] \equiv x_{r}\left[x_{j}, x_{n}\right]\left[x_{1}, x_{s}\right]-x_{s}\left[x_{j}, x_{n}\right]\left[x_{1}, x_{r}\right]$ this case is a consequence of the previous cases.

Now let us consider $r, s, k, l \in\{2, \cdots, n\}$. In this case the result follows since

$$
\begin{aligned}
P_{k, l, r, s} & \equiv w^{\prime} x_{1}\left[x_{k}, x_{l}\right]\left[x_{r}, x_{s}\right] \\
& \equiv w^{\prime} x_{k}\left[x_{1}, x_{l}\right]\left[x_{r}, x_{s}\right]-w^{\prime} x_{l}\left[x_{1}, x_{k}\right]\left[x_{r}, x_{s}\right] .
\end{aligned}
$$

Analogously, the result can be proved for the polynomials $\tilde{P}_{k, l, r, s}$.

By using (2) and (5), induction on $n$ and analogous reasoning as in the Lemma 2.4 we have the next result. Its proof will be omited here.

Lemma 2.5. For all $n \geq 4$, any polynomial of the form $w\left(x_{r}\left[x_{k}, x_{l}\right] x_{s}-\right.$ $\left.x_{r} x_{s}\left[x_{k}, x_{l}\right]\right)$ can be written, modulo $Q$, as a linear combination over $F$ of polynomials in $T(I I) \cup T(I V)$, where $l, r, s, k \in\{1, \cdots, n\}$ and $w$ is the product of remaining ordered variables $x_{i}$ with $i \neq l, r, s, k$.

Our last lemma is the crucial tool in the proof of Theorem 2.2.

Lemma 2.6. For all $n \geq 4$, any polynomial of the form $w\left[x_{r}, x_{s}\right]$ can be written, modulo $Q$, as a linear combination over $F$ of elements in $T(I I) \cup$ $T(I V)$, where $r, s \in\{1, \cdots, n\}$ and $w$ is the ordered product of the variables $x_{i}$ with $i \neq r, s$.

Proof. By Lemmas 2.4 and 2.5, it is enough to prove that $w\left[x_{r}, x_{s}\right]$ can be written, modulo $Q$, as a linear combination over $F$ of polynomials of 
the types $P_{k, l, r, s}, \tilde{P}_{k, l, r, s}$ and $w^{\prime}\left(x_{r}\left[x_{k}, x_{l}\right] x_{s}-x_{r} x_{s}\left[x_{k}, x_{l}\right]\right)$, where $r, s, k, l \in$ $\{1, \cdots, n\}$.

By using (1), that $f_{3} \in Q$ and some calculations we have

$$
\begin{aligned}
4 x y[w, z] \equiv & -2(x[w, z] y-x y[w, z])-(w x[z, y]-w[z, y] x) \\
& -(w y[z, x]-w[z, x] y)-(z x[y, w]-z[y, w] x) \\
& -(z y[x, w]-z[x, w] y)+[x, y][z, w] \\
& +2[w, z][y, x]+2[y, w][z, x]+2[z, y][w, x] .
\end{aligned}
$$

Now since $w\left[x_{r}, x_{s}\right]=w^{\prime} x_{k} x_{l}\left[x_{r}, x_{s}\right]$, the computations above guarantee the result. See details in [8].

At this point we are ready to prove the most important result of this section.

\section{Proof of the Theorem 2.2.}

Observe that $Q \subset \operatorname{Id}\left(M_{7}\right)$. As $f_{7}=[x, y][z, w][t, v] \in Q$, by a well known fact (see [1] Theorem 5.2.1) we have that any multilinear polynomial of degree $n$ can be written, modulo $Q$, as a linear combination of polynomials of the type

$x_{i_{1}} \cdots x_{i_{m}}\left[x_{j}, x_{j_{1}}, \cdots, x_{j_{q}}\right]\left[x_{k}, x_{k_{1}}, \cdots, x_{k_{p}}\right]$
where $j_{1}<\cdots<j_{q}, \quad k_{1}<\cdots<k_{p}, j>j_{1}, \quad k>k_{1}$ and $i_{1}<\cdots<i_{m}$.

We shall use this fact to find a generating set for $\frac{P_{n}}{P_{n} \cap Q}$.

We note that if $c$ is a left normed commutator of arbitrary weight, by using the identities $f_{4}$ and $f_{5}$ we have

$$
\begin{gathered}
{[x, y, z] c \equiv[x, y] z c-z[x, y] c \equiv-z[x, y] c} \\
c[x, y, z] \equiv c[x, y] z-c z[x, y] \equiv c[x, y] z \equiv z c[x, y] .
\end{gathered}
$$

Therefore, it is enough to consider polynomials of the following types

$$
\begin{gathered}
x_{i_{1}} \cdots x_{i_{m}}\left[x_{j}, x_{j_{1}}\right]\left[x_{k}, x_{k_{1}}\right] \\
x_{i_{1}} \cdots x_{i_{m}}\left[x_{j}, x_{j_{1}}, \cdots, x_{j_{n-m-1}}\right]
\end{gathered}
$$

where $j_{1}<\cdots<j_{n-m-1}, \quad j>j_{1}, \quad k>k_{1}, \quad i_{1}<\cdots<i_{m}$ and $m \neq n-1$.

On the other hand, commutators of weight greater or equal to 3 can be rewritten in terms of commutators of weight 2 by considering the remaining variables outside of the commutator, for example:

$$
\begin{aligned}
{[x, y, z, w, t]=} & ([x, y] z w t-z[x, y] w t)-(w[x, y] z-w z[x, y]) t \\
& +(t[x, y] z-t z[x, y]) w-t(z[x, y] w-z w[x, y]) .
\end{aligned}
$$

Thus, for $m \neq n$, any polynomial in (6) and in (7) is a linear combination over $F$ of polynomials of the type

$$
x_{i_{1}} \cdots x_{i_{m}}\left[x_{j}, x_{j_{1}}\right]\left[x_{r}, x_{r_{1}}\right]
$$




$$
\begin{gathered}
x_{i_{1}} \cdots x_{i_{m}}\left[x_{j}, x_{j_{1}}\right] \\
x_{i_{1}} \cdots x_{i_{m}}\left(\left[x_{j}, x_{j_{1}}\right] x_{j_{2}} \cdots x_{j_{n-m-1}}-x_{j_{2}}\left[x_{j}, x_{j_{1}}\right] x_{j_{3}} \cdots x_{j_{n-m-1}}\right) \\
x_{i_{1}} \cdots x_{i_{n-4}}\left(x_{r}\left[x_{j}, x_{j_{1}}\right] x_{s}-x_{r} x_{s}\left[x_{j}, x_{j_{1}}\right]\right) \\
x_{i_{1}} \cdots x_{i_{n-5}}\left(x_{r}\left[x_{j}, x_{j_{1}}\right] x_{s}-x_{r} x_{s}\left[x_{j}, x_{j_{1}}\right]\right) x_{k},
\end{gathered}
$$

with $i_{1}<\cdots<i_{m}, j>j_{1}$ and $r>r_{1}$.

By the previous constructions and lemmas, in order to show that

$$
X=T(I) \cup T(I I) \cup T(I I I) \cup T(I V)
$$

generates $P_{n}$, module $P_{n} \cap Q$, it is enough to prove that a polynomial of the form $w^{\prime}\left(x_{k}\left[x_{r}, x_{s}\right] x_{l}-x_{k} x_{l}\left[x_{r}, x_{s}\right]\right) x_{t}$, with $k, l, r, s, t \in\{1, \cdots, n\}$, is a linear combination of polynomials in $X$. This follows from

$$
w^{\prime}\left(x_{k}\left[x_{r}, x_{s}\right] x_{l}-x_{k} x_{l}\left[x_{r}, x_{s}\right]\right) x_{t} \equiv w^{\prime}\left(x_{t}\left[x_{l}, x_{k}\right]\left[x_{r}, x_{s}\right]+x_{k}\left[x_{r}, x_{s}\right]\left[x_{l}, x_{t}\right]\right) \text {. }
$$

Now we note that

$$
|T(I)|=1,|T(I I)|=|T(I I I)|=n-1
$$

and

$$
|T(I V)|=5+2 \sum_{j=5}^{n}(j-2)=n^{2}-3 n+1 .
$$

This implies that

$$
|X|=|T(I)|+|T(I I)|+|T(I I I)|+|T(I V)|=n^{2}-n .
$$

Therefore, if $V=\operatorname{var}(Q)$ we have $c_{n}\left(M_{7}\right) \leq c_{n}(V) \leq n^{2}-n$.

If $\lambda=(n)$, the corresponding highest weight vector $f_{T_{\lambda}}=x^{n} \notin \operatorname{Id}\left(M_{7}\right)$ because $f_{T_{\lambda}}\left(e_{11}+e_{33}\right)=e_{11}+e_{33} \neq 0$. Then $m_{(n)} \neq 0$.

On the other hand, if $\lambda=(n-1,1)$ then, by Remark 1.4 , we affirm that $m_{\lambda} \geq 2$. Indeed let

$$
f_{T_{\lambda}^{(n)}}=x_{1}^{n-1} x_{2}-x_{2} x_{1}^{n-1} \text { and } f_{T_{\lambda}^{(n-1)}}=x_{1}^{n-2} x_{2} x_{1}-x_{2} x_{1}^{n-1}
$$

be the highest weight vectors corresponding to standard tableaus $T_{\lambda}^{(n)}$ and $T_{\lambda}^{(n-1)}$ with $n$ and $n-1$ in the only box of the second line respectively, that is,

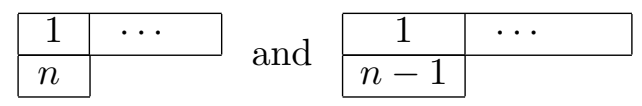

We have that $f_{T_{\lambda}^{(n)}}, f_{T_{\lambda}^{(n-1)}} \notin I d\left(M_{7}\right)$ and are linearly independent modulo $\operatorname{Id}\left(M_{7}\right)$ since

$$
f_{T_{\lambda}^{(n)}}\left(e_{11}+e_{33}, e_{12}\right)=e_{12} \neq 0 \text { and } f_{T_{\lambda}^{(n-1)}}\left(e_{11}+e_{33}, e_{23}\right)=-e_{23} \neq 0
$$


and also

$$
f_{T_{\lambda}^{(n-1)}}\left(e_{11}+e_{33}, e_{12}\right)=0 .
$$

Now if $\lambda=(n-2,1,1)$ then the corresponding highest weight vector

$$
f_{\lambda}=S t_{3}\left(x_{1}, x_{2}, x_{3}\right) x_{1}^{n-3} \notin \operatorname{Id}\left(M_{7}\right)
$$

since $f_{\lambda}\left(e_{11}+e_{33}, e_{12}, e_{23}\right)=2 e_{13} \neq 0$. Hence $m_{(n-2,1,1)} \neq 0$.

Finally when $\lambda=(n-2,2)$, we get that the highest weight vector

$$
f_{\lambda}=x_{1} x_{2} x_{1} x_{2} x_{1}^{n-4}-x_{1} x_{2}^{2} x_{1}^{n-3}-x_{2} x_{1}^{2} x_{2} x_{1}^{n-4}+x_{2} x_{1} x_{2} x_{1}^{n-3}
$$

does not belong to $\operatorname{Id}\left(M_{7}\right)$ because $f_{\lambda}\left(e_{11}+e_{33}, e_{12}+e_{23}\right)=-e_{13} \neq 0$. Therefore $m_{(n-2,2)} \neq 0$.

It follows that

$c_{n}\left(M_{7}\right) \geq \operatorname{deg} \chi_{(n)}+2 \operatorname{deg} \chi_{(n-1,1)}+\operatorname{deg} \chi_{(n-2,1,1)}+\operatorname{deg} \chi_{(n-2,2)}=n(n-1)$, where the last equality comes from the hook formula. Thus

$$
n(n-1) \leq c_{n}\left(M_{7}\right) \leq c_{n}(V) \leq n(n-1),
$$

and so $c_{n}\left(M_{7}\right)=n(n-1)$ and $I d\left(M_{7}\right)=Q$. Therefore, $X$ is a basis for $\frac{P_{n}}{P_{n} \cap \operatorname{Id}\left(M_{7}\right)}$ and $\chi_{n}\left(M_{7}\right)=\chi_{(n)}+2 \chi_{(n-1,1)}+\chi_{(n-2,1,1)}+\chi_{(n-2,2)}$. Consequentely, $l_{n}\left(M_{7}\right)=5$ and the theorem is proved.

\section{Minimal varieties of quadratic growth}

Giambruno and La Mattina also proved ([3], Corollary 14 and Theorem 22 ) the next two results. In the first of them the authors characterized the algebras with constant growth of their identities while in the second one they do the same for algebras with linear growth of identities.

Theorem 3.1. Given an F-algebra $A$, the algebras $M_{1}, M_{2}, M_{3} \notin \operatorname{var}(A)$ if and only if $c_{n}(A) \leq k$ for some constant $k$, for all $n \geq 1$.

Theorem 3.2. Let $A$ be an F-algebra. Then $M_{3}, M_{4}, M_{5}, M_{6}, M_{7} \notin$ $\operatorname{var}(A)$ if and only if $c_{n}(A) \leq k n$ for some constant $k$, for all $n \geq 1$.

Next we classify (up to PI-equivalence) on minimal varieties quadratic growth, i.e., varieties with the following property.

Definition 3.3. Given an algebra $\mathcal{A}$ we say that the variety of algebras $V=\operatorname{var}(\mathcal{A})$ is minimal of quadratic growth if the sequence of codimensions $c_{n}(V)=c_{n}(\mathcal{A})$ has quadratic growth and all proper subvariety of $V$ has linear or constant growth. 
In order to obtain this classification, note that if $\operatorname{var}(A)$ denotes the variety of algebras generated by $A$ and $B$ is a $F$-algebra such that $B \in$ $\operatorname{var}(A)$, then $\operatorname{Id}(A) \subset I d(B)$, hence $P_{n}(B)$ can be embedded in $P_{n}(A)$. Therefore, $c_{n}(B) \leq c_{n}(A)$ and $l_{n}(B) \leq l_{n}(A)$, for all $n \geq 1$.

On the other hand, by Theorem 2.2 and Lemma 2.1 we observe that the algebras $M_{i}$, for $3 \leq i \leq 7$, have quadratic codimension growth. We can also prove that they generate the only minimal varieties of quadratic growth.

Theorem 3.4. The varieties $\operatorname{var}\left(M_{3}\right), \operatorname{var}\left(M_{4}\right), \operatorname{var}\left(M_{5}\right), \operatorname{var}\left(M_{6}\right)$, and $\operatorname{var}\left(M_{7}\right)$ are minimal of quadratic growth.

Proof. Let $W=\operatorname{var}(A)$ be a proper subvariety of $\operatorname{var}\left(M_{7}\right)$ where $A$ is a $F$-algebra. Then $\operatorname{Id}\left(M_{7}\right) \varsubsetneqq \operatorname{Id}(A), M_{7} \notin W$ and

$$
c_{n_{0}}(A) \supsetneqq c_{n_{0}}\left(M_{7}\right) \text {, for some natural } n_{0} \text {. }
$$

Moreover

(1) $M_{2}, M_{3} \notin W$, since

$$
f=[[x, y],[z, x]]+y x[x, z]+z x[y, x]+x^{2}[z, y] \in \operatorname{Id}\left(M_{7}\right) \varsubsetneqq \operatorname{Id}(A),
$$

but $f \notin \operatorname{Id}\left(M_{2}\right)$ and $\notin I d\left(M_{3}\right)$ because $f \not \equiv 0$ in $M_{2}$ and in $M_{3}$.

(2) $M_{4}, M_{5}, M_{6} \notin W$ otherwise if $M_{i} \in W$ for some $i=4,5,6$ then $\operatorname{Id}(A) \subseteq \operatorname{Id}\left(M_{i}\right)$ and by Lemma 2.1 we have

$$
c_{n_{0}}\left(M_{7}\right)=c_{n_{0}}\left(M_{i}\right) \leqq c_{n_{0}}(A) \supsetneqq c_{n_{0}}\left(M_{7}\right)
$$

what is a contradiction.

Now if $M_{1} \notin W$ then by Theorem 3.1 it follows that $c_{n}(A)=c_{n}(W)$ has constant growth. Otherwise, by Theorem 3.2 we conclude that $c_{n}(A)=$ $c_{n}(W)$ has linear growth and the result is proved for $\operatorname{var}\left(M_{7}\right)$.

Now if $W=\operatorname{var}(A)$ is a proper subvariety of $\operatorname{var}\left(M_{3}\right)$ we have $M_{i} \notin W$ for $2 \leq i \leq 7$. In fact, $M_{3} \notin W$ and

$$
f=[x, y, z] \in \operatorname{Id}\left(M_{3}\right) \varsubsetneqq \operatorname{Id}(A) \text { but } f \not \equiv 0 \text { in } M_{i} \text {, when } i=2,4,5,6,7 \text {. }
$$

Consequentely $W$ has linear or constant growth according to $M_{1}$ belongs or not or not to $W$, respectively.

On the other hand, if $U=\operatorname{var}(B)$ is a proper subvariety of $\operatorname{var}\left(M_{4}\right)$ we have $M_{4} \notin U$ and since $c_{n}\left(M_{4}\right)=c_{n}\left(M_{5}\right)=c_{n}\left(M_{6}\right)=c_{n}\left(M_{7}\right)$ we use the same idea of item 2 above to conclude that $M_{5}, M_{6}, M_{7} \notin U$.

Now since $f=[x, y] z w \in \operatorname{Id}\left(M_{4}\right) \varsubsetneqq \operatorname{Id}(B)$ but $f \notin \operatorname{Id}\left(M_{3}\right) \cup \operatorname{Id}\left(M_{1}\right)$ we have that $M_{1}, M_{3} \notin U$. Then $U$ has linear or constant growth depending on $M_{2}$ belonging or not to $U$, respectively. 
We use that $f=z w[x, y] \in I d\left(M_{5}\right)$ and $g=z[x, y] w \in I d\left(M_{6}\right)$ and a similar reasoning as before to prove that all proper subvarieties of $\operatorname{var}\left(M_{5}\right)$ and $\operatorname{var}\left(M_{6}\right)$ have linear or constant growth and this proves the theorem.

Corollary 3.5. The varieties $\operatorname{var}\left(M_{3}\right), \operatorname{var}\left(M_{4}\right), \operatorname{var}\left(M_{5}\right), \operatorname{var}\left(M_{6}\right)$ and $\operatorname{var}\left(M_{7}\right)$ are the only minimal varieties of quadratic growth.

\section{References}

[1] V. Drensky, Homogeneous polynomial identities, Israel J. Math. 42 (1982) 258272.

[2] V. Drensky, Free Algebras and PI-Algebras. Springer-Verlag Singapore (2000).

[3] A. Giambruno \& D. La Mattina, PI-algebras with slow codimension growth, $J$. Algebra 284 (2005) 371-391.

[4] A. R. Kemer, T-ideals with power growth of the codimensions are Specht, Sibirsk. Math. Zh. 19 (1978) 54-69 (in Russian). English translation in: Siberian Math. J. 19 (1978) 37-48.

[5] G. James \& A. Kerber, The representation theory of the symmetric group. London: Addison-Wesley Publishing Company (1981).

[6] S. Mishchenko, A. Regev, M. Zaicev, A characterization of PI algebras with bounded multiplicities of the cocharacters, J. Algebra 219 (1999) 356-368.

[7] A. Regev, Existence of identities in $A \otimes B$, Israel J. Math. 11 (1972) 131-152.

[8] A. C. Vieira \& S. M. Alves Jorge, On Minimal Varieties of Quadratic Growth, Linear Algebra and its Applications. To appear. 